\title{
Burnout consequences in caregivers of patients with dementia
}

\author{
Ciobanu Adela Magdalena ${ }^{1,2}$,Prelipcean Laurentiu Nicolae ${ }^{2}$, Niculae Carmen Petrina ${ }^{2}$, \\ Velcea Diana ${ }^{2}$
}

\author{
${ }^{1}$ Associated Professor Dr. "University of Medicine and Pharmacy Carol Davila" \\ Bucharest, Romania \\ ${ }^{2}$ Clinical Department, Bucharest, Romania
}

\section{OBJECTIVES}

The purposes of this study are outlining an inversely proportional relationshionship between MMSE scores for the dementia diagnosed patients and the severity of burn-out symptoms among caregivers directly involved in looking after dementia diagnosed patients.

\section{BACKGROUND AND AIMS}

Studies have shown that more than half of the caregivers for the dementia diagnosed patients develop burnout symptoms. Our aim is to highlight the association between burnout symptoms gravity and cognitive impairment and also a directly proportional relation between the severity of burn-out symptoms and kinship degree. [1]

\section{MATERIALS AND METHODS}

In this study we have included individuals with negative psychiatric background, active professional status and socioeconomically satisfactory status, and we excluded individuals with preexistent psychiatric disorders. We have selected a group of 50 dementia diagnosed patients, in various evolutive stages, and 75 caregivers. We have applied Maslach Scale for evidencing the presence and the severity of burnout syndrome. The study has been conducted over a 24 months period. [2]

\section{RESULTS}

Our findings show that 45 of the caregivers have addressed emergency medical services, as follows:

$>12$ of them were diagnosed with exacerbation of preexistent somatic diseases,

$>28$ of them were diagnosed both with psychiatric and somatic disorders,

$>5$ of them passed away.

$>$ All of the caregivers experienced burnout symptoms of various degrees.

\section{CONCLUSIONS}

Burnout syndrome is worsened in direct proportion to the decrease in patient self-care capacity, which leads to decreased quality of life for both. It is difficult for caregivers to divide between family life, profession, recreational / daily activities and the act of care. Also, caring for a person with dementia is a strong risk factor for developing depression, anxiety, decompensation of somatic diseases and psycho-somatic diseases; this risk increases if the caregiver's physical disabilities are added. The MMSE scores are inversely proportional to the Maslach scores of the carriers. Directly proportional to the increase in the severity of dementia and inability to care, the intensity of feelings of burden and burnout syndrome increases in people in care. [3]

\section{Burnout consequences}

Studies show that burnout syndrome symptoms are a result of inefficient coping mechanisms in the face of stress. For example, a study shows that 111 caregivers were evaluated, and 37 of them presented chronic symptoms (emotional exhaustion, physical and mental exhaustion), lasting at least 6 months. They had high levels of stress in the workplace, irritability after program termination, sleep disturbances, daytime sleepiness and increased levels of serum cortisol throughout the day. These data suggest a link between burnout syndrome and cardio-vascular pathology.

Preventing as also as an early diagnosis of burnout syndrome for the caregivers reduces the incidence of various psychiatric pathologies. Psychotherapy and counseling for the caregivers are of a significant importance. [4]

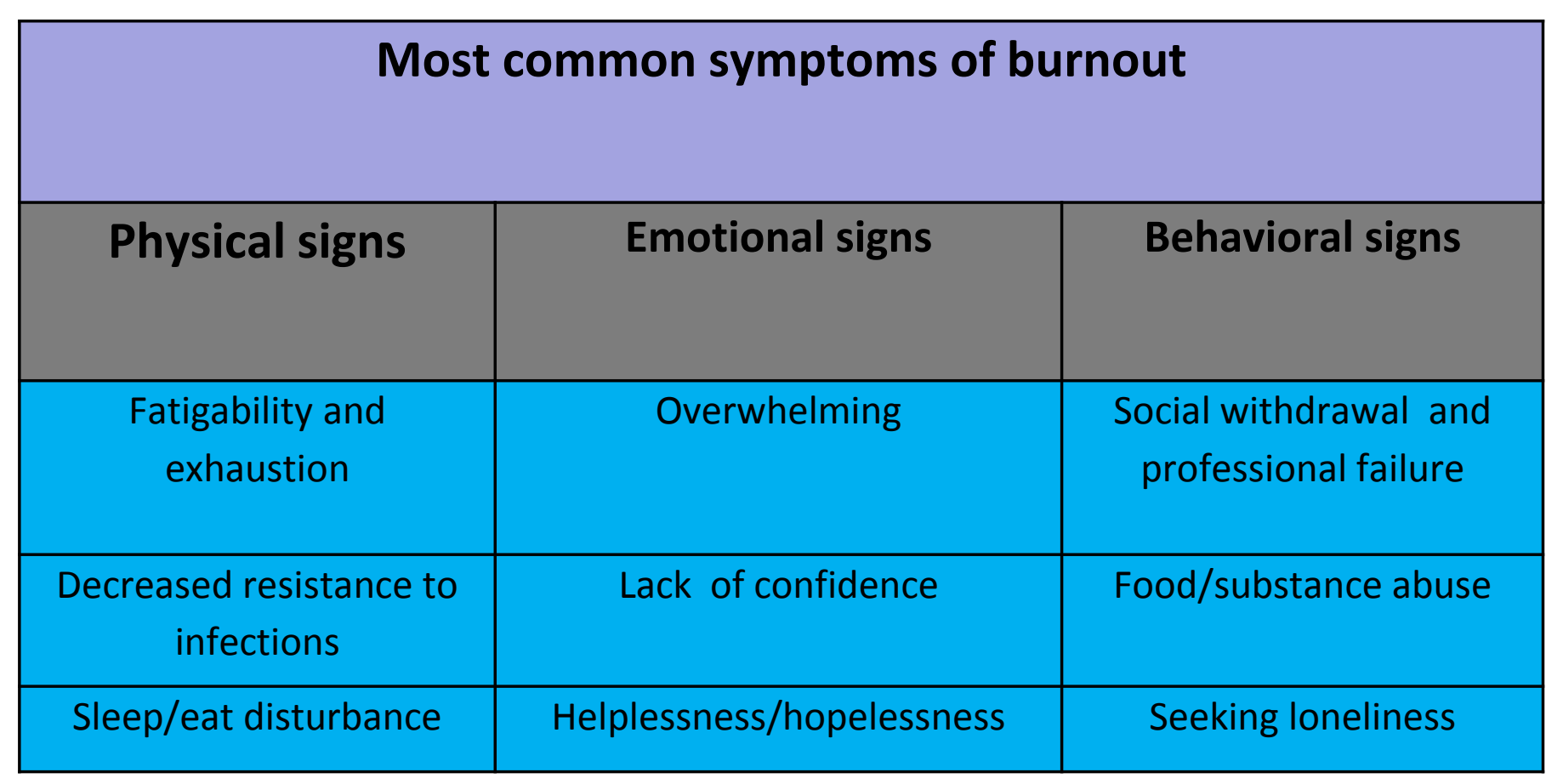

